\title{
Plasmodium Vivax and Dengue co Infection Emerging Challenge to Diagnosis
}

\author{
Rateesh Sareen* and Menka Kapil \\ Department of Pathology \& Transfusion Medicine, Santokba Durlabhji Hospital, Jaipur. (India)
}

\begin{abstract}
The prevalence of malaria and dengue is high in tropical countries. The diagnosis of mixed infections is difficult as both infections have similar clinical presentation. There is paucity of literature on concurrent malaria and dengue infections. The confirmation of one confection should not preclude the possibility of other as mixed infections have poor prognostic outcome. The timely diagnosis of co infection is important for favorable outcome and complete cure.
\end{abstract}

Keywords: Malaria, Dengue, Concurrent infection

\section{Introduction}

There are several tropical mosquito borne infection. Malaria and Dengue are the disease caused by mosquito which is very common in India. Malaria is a parasitic disease transmitted by Anopheles mosquito and Dengue is a viral disease which is transmitted by Aedes mosquito Seldom reports have been published on concurrent infection by malaria and dengue $e^{1,2}$. Co-existing infections (Malaria and dengue) go undetected due to lack of clinical suspicion and overlapping symptoms. Co-existing infections are on the rise in tropics and cause high morbidity and mortality around the world. WE report a case of concurrent malaria and dengue to highlight that physicians in endemic region should be vigilant for the possibility of concurrent malaria and dengue infection.

\section{Case Report}

In the present case, 35 year old woman was suffering from high grade fever with chills and rigors and with abdominal pain. She was given two units of whole blood donations in a district hospital but her fever did not improve. She was subsequently brought to tertiary care hospital at Jaipur. On admission her general physical examinations revealed fever 100.8 degree $\mathrm{F}$, pulse rate $112 / \mathrm{min}$ and blood pressure $110 / 70 \mathrm{~mm}$ of $\mathrm{Hg}$. There was palpable liver but no splenomegaly. Personal and past history was unremarkable.

The laboratory investigations revealed-BUN- $13 \mathrm{mg} / \mathrm{dl}$, Creatinine $-0.4 \mathrm{mg} / \mathrm{dl}$, Sodium $132 \mathrm{~m} \mathrm{~mol} /$ litre, potassium $3.4 \mathrm{~m} \mathrm{~mol} /$ liter, chloride $104 \mathrm{~m} \mathrm{~mol} / \mathrm{lit}$, glucose $134 \mathrm{mg} /$ dl, SGOT- 43U/L,SGPT $48 \mathrm{U} / \mathrm{L}$, total Bilirubin $2.4 \mathrm{mg} /$ dl, Direct Bilirubin-0.9 mg/dl, Total protein-6.1 gm/dl ,Albumin $2.7 \mathrm{gm} / \mathrm{dl}$, globulin $3.4 \mathrm{gm} / \mathrm{dl}$ [ inverse] A/G ratio 0.79,Alkaline Phoshatase 126U/L, Gamma GT-
25U/L, bleeding time was 2 minutes, clotting time- 5 minutes.PT13.6 seconds, INR was 1.00. Complete blood count examination results are enumerated in table-1.

On the day of admission her rapid Malaria test was positive for Plasmodium vivax and negative for Plasmodium falciparum antigen by Rapid card detection [Sure test PF/ PV HRP2/PLDH COMBO].This finding was confirmed by presence of schizonts of P.vivax on peripheral blood smear ( Figure -1). Widal test was negative. She was reported as positive for IgM dengue antibody while Ig $\mathrm{G}$ and antigen were negative on ELISA. The patient was diagnosed as a case of concurrent malaria and dengue infection. She was treated with intra venous fluid, oral chloroquine, artesunate and antibiotics. On second day she was improved clinically as became a febrile and improvement of platelets count. Repeated peripheral smears were negative for plasmodium. The patient was recovered completely and discharged on sixth day.

\section{Discussion:}

Malaria and dengue fever are prevalent vector-borne diseases worldwide and represent major public health problems. Dengue epidemics have been reported in several countries; 500,000 people with severe dengue require hospitalization each year, and $2.5 \%$ of those affected die. Similarly, malaria is a life-threatening disease which was responsible for 627,000 deaths in $2012^{[3,4]}$. Concurrent infection with these two different infective agents causes overlapping of clinical features thus leads to a diagnostic challenge for physicians .Many studies shows that co infection may be more severe as compared to single infection ${ }^{[5]}$ The first case of concurrent dengue and Plasmodium falciparum was published by Charrel et al. in $2005 .{ }^{[6]}$ Malaria and dengue fever are prevalent in 
TABLES:

\begin{tabular}{|c|c|c|c|c|}
\hline & DAY 1 & DAY 2 & DAY 3 & DAY 4 \\
\hline R B C ( x 109 / mm3) & 2.81 & 2.93 & 2.76 & 3.59 \\
\hline $\mathrm{H} \mathrm{b}(\mathrm{g} / \mathrm{dL})$ & 7.1 & 7.4 & 6.8 & 9.6 \\
\hline Hct $(\%)$ & 22.3 & 23.1 & 21.7 & 30.3 \\
\hline WBC ( $\left.\times 10^{3} / \mathrm{mm} 3\right)$ & 5.03 & 3.81 & 5 & 6.53 \\
\hline NEUTRO ( x 10³/ mm3) & 3.32 & 1.77 & 2.39 & 4 \\
\hline LYMPH ( x 10³ / mm3) & 1.47 & 1.94 & 2.38 & 2.04 \\
\hline EOSINO ( x 103/ mm3) & 0.12 & 0.02 & 0.07 & 0.23 \\
\hline MONOCYTE ( x 103 / mm3) & 0.11 & 0.07 & 0.15 & 0.25 \\
\hline BASOPHIL ( x 103 / mm3) & 0.01 & 0.01 & 0.01 & 0.01 \\
\hline IG $\left(\times 10^{3} / \mathrm{mm} 3\right)$ & 0.15 & 0.23 & 0.46 & 0.68 \\
\hline PLATELETS ( $\left.\times 10^{9} / \mathrm{mm} 3\right)$ & 120 & 135 & 150 & 150 \\
\hline
\end{tabular}

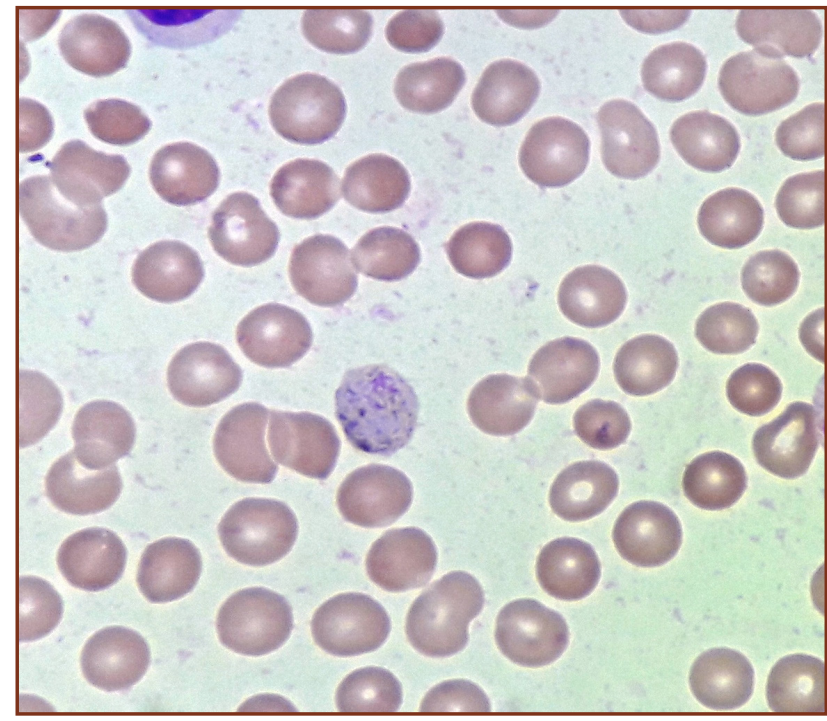

Fig.1: PBF Leishman stain ( 100 x) showing Malaria parasite

tropical countries. The probability of malaria and dengue coinfection in the same patient is quiet rare as both the illness differ in certain respects. Malaria, a protozoa infection, is caused by bite of Anopheles mosquito whereas dengue is a viral infection transmitted to humans by bite of infected Aedes aegypti. Dengue mosquito is considered a day-biting mosquito, while malaria is a night-biting mosquito. Dengue is more prevalent in urban areas whose reservoir is stored clean water, while malaria is seen in rural areas breeding in stagnant water sources. Hence, overlapping habitat may not be easily available and concurrent infection has to be by chance. ${ }^{[7]}$ one of the reason of concurrent infection may be because of few cases of P.Vivax at the liver stage of the parasite become asymptomatic after the primary attack. During the long latent stage, asymptomatic carriers show dual infection by P.vivax and other pathogens. ${ }^{[8]}$
The differentiation of malaria from dengue based solely on clinical grounds is difficult, specific diagnostic testing is required to confirm diagnosis. As reinforced by our case report the confirmation of one confection should not preclude the possibility of confection. The case report $s$ from literature have proven that delayed diagnosis have resulted in fatal outcome, therefore for a better prognosis treatment should be instituted as early as possible ${ }^{[9,10]}$

\section{Conclusion}

In areas that are endemic for malaria and dengue there is increasing trend of concurrent infection therefore one should always be vigilant while treating single infection as concurrent infection have poor prognosis if left untreated.

\section{Acknowledgements \\ DR G N GUPTA HEAD OF THE DEPARTMENT, SDMH HOSPITAL, JAIPUR}

\section{Reference}

1. Charrel RN, Brouqui P, Foucault C, de Lamballerie X. Concurrent dengue and malaria. Emerg Infect Dis 2005; 11:1153-4.

2. Arya SC, Mehta LK, Agarwal N, Agarwal BK, Mathai G, Moondhara A. Episodes of concurrent dengue and malaria. Dengue Bull 2005; 29:208-9.

3. World Health Organization (2010) World Malaria Report. Geneva, Switzerland.

4. WHO (2009) Dengue: Guidelines for diagnosis, treatment, prevention and control. Geneva, Switzerland.

5. Epelboin, M. Hanf, P. Dussart et al., "Is dengue and malaria co-infection more severe than single infections? A retrospective matched-pair study in French Guiana," Malaria Journal, vol. 11, p. 142, 2012.

6. R. N. Charrel, P. Brouqui, C. Foucault, and X. De Lamballerie, "Concurrent dengue and malaria,"Emerging Infectious Diseases, vol. 11, no. 7, pp. 1153-1154, 2005. 
7. Wiwanitkit V. Concurrent malaria and dengue infection: A brief summary and comment. Asian Pac J Trop Biomed. 2011;1:326-7.[PMC free article] [PubMed]

8. P.S.Thangratham, M.K.Jeevan, R.Rajendran, P.Philip Samuel and B.K. Tyagi. Dual infection by Dengue virus and Plasmodium vivax in A lappuazha district, kerala, India. Jpn.J.Infect.Dis,59,2006

9. Ali N, Nadeem A, Anwar M, Tariq WZ, Chotani RA..
Dengue fever in malaria endemic areas. J Coll Physicians Surg Pak 2006; 16:340-2.

10. 10]Ward DI. A case of fatal Plasmodium falciparum malaria complicated by acute dengue fever in East Timor. Am J Trop Med Hyg 2006; 75:182-5

11. Janis EB. Evaluation of fever in the international traveler. Unwanted 'souvenir' can have many causes. Postgrad Med 2004; 116:13-29.

*Corresponding author:

Dr Rateesh Sareen, D.N.B Pathology. Department of Pathology \& Transfusion Medicine, Santokba Durlabhji Hospital, Jaipur. (India)

Phone: +9101412566251

Email: drrateeshsareen@yahoo.co.in.

Financial or other Competing Interests: None. 